\title{
Electron-Electron Interaction in Strong Electromagnetic Fields: The Two-Electron Contribution to the Ground-State Energy in He-Like Uranium
}

\author{
A. Gumberidze ${ }^{1,2,3}$, Th. Stöhlker ${ }^{1,2}$, D. Banaś ${ }^{4}$, K. Beckert ${ }^{1}$, P. Beller ${ }^{1}$, \\ H.F. Beyer ${ }^{1}$, F. Bosch ${ }^{1}$, X. Cai ${ }^{5}$, S. Hagmann ${ }^{1,2}$, C. Kozhuharov ${ }^{1}$, \\ D. Liesen ${ }^{1}$, F. Nolden ${ }^{1}$, X. Ma ${ }^{5}$, P.H. Mokler ${ }^{1}$, A. Oršić-Muthig ${ }^{1,2}$, \\ M. Steck ${ }^{1}$, D. Sierpowski ${ }^{6}$, S. Tashenov ${ }^{1,2}$, A. Warczak $^{6}$ and Y.Zou ${ }^{7}$ \\ ${ }^{1}$ Gesellschaft für Schwerionenforschung 64291 Darmstadt Germany \\ ${ }^{2}$ Institut für Kernphysik University of Frankfurt 60486 Frankfurt Germany \\ ${ }^{3}$ Tbilisi State University Tbilisi Georgia \\ ${ }^{4}$ Institute of Physics Swietokrzyska Academy 25-406 Kielce Poland \\ ${ }^{5}$ Institute of Modern Physics 730000 Lanzhou China \\ ${ }^{6}$ Institute of Physics Jagiellonian University 30-059 Cracow Poland \\ ${ }^{7}$ Fudan University, Shanghai, China
}

\begin{abstract}
Radiative recombination transitions into the ground state of cooled bare and hydrogen-like uranium ions were measured at the storage ring ESR. By comparing the corresponding x-ray centroid energies, this technique allows for a direct measurement of the electron-electron contribution to the ionization potential in the heaviest He-like ions. For the two-electron contribution to the ionization potential of He-like uranium we obtain a value of $2248 \pm 9 \mathrm{eV}$. This represents the most accurate determination of two-electron effects in the domain of high-Z He-like ions and the accuracy reaches already the size of the specific two-electron radiative QED corrections.
\end{abstract}


Helium-like ions are the simplest and most fundamental atomic multi-body systems. Structure investigations of these systems along the isoelectronic sequence up to the heaviest species uniquely probe our understanding of correlation, relativistic, and quantum electrodynamical effects. Compared to low-Z ions where binding energies are affected most significantly by the non-QED contributions to the electron-electron interaction, the situation is distinctively different for He-like ions at high Z. At such conditions the bound electrons are exposed to extreme nuclear Coulomb fields of about $10^{16} \mathrm{~V} / \mathrm{cm}$ which are close to the critical field strength where spontaneous electron-positron production is expected to occur [1]. These strong external fields significantly alter the electron-electron interaction of the two strongly bound electrons via relativistic and quantum electrodynamical effects $[2,3]$. As a consequence it is expected that correlation and the specific QED corrections for the electron-electron interaction are of the same order of magnitude providing a challenge for theoretical description.

Recently, a significant progress took place in theory, where a new generation of relativistic many-body calculations has established improved benchmarks for the non-QED part of the electron-electron interaction [4-7]. For the groundstate the progress achieved is particularly impressive since even the specific two-electron radiative QED corrections can nowadays be calculated without any approximations and all the two-photon exchange diagrams for the electron-electron interaction are considered in a complete fashion $[2,3]$. As a consequence, the theoretical accuracy for the electron-electron contribution to the ground-state ionization potential of He-like ions at high-Z is claimed to be comparable or even better than the one currently available for the one-electron part. Therefore the ground state of high-Z Helike ions is of particular relevance for probing bound-state QED in strong fields since to date, the two-electron contribution to the ionization potential in He-like systems is the only measurable value which has been calculated completely to second order in $\alpha$ [8].

In this letter we report a first measurement of the two-electron contribution to the ionization potential for the heaviest ion available to experiments, i.e. He-like uranium. The experiment, conducted at the ESR (Experimental Storage Ring) at GSI-Darmstadt, is based on a novel experimental technique introduced at the Super-EBIT device which exploits radiative recombination (RR) transitions into the vacant $K$-shell of $\mathrm{H}$ - and He-like high- $Z$ ions (Fig. 1)[9]. This allowed us to measure the ionization potential of the He-like species with respect to the $\mathrm{H}$-like ions and to determine the two-electron contribution to the ground state 


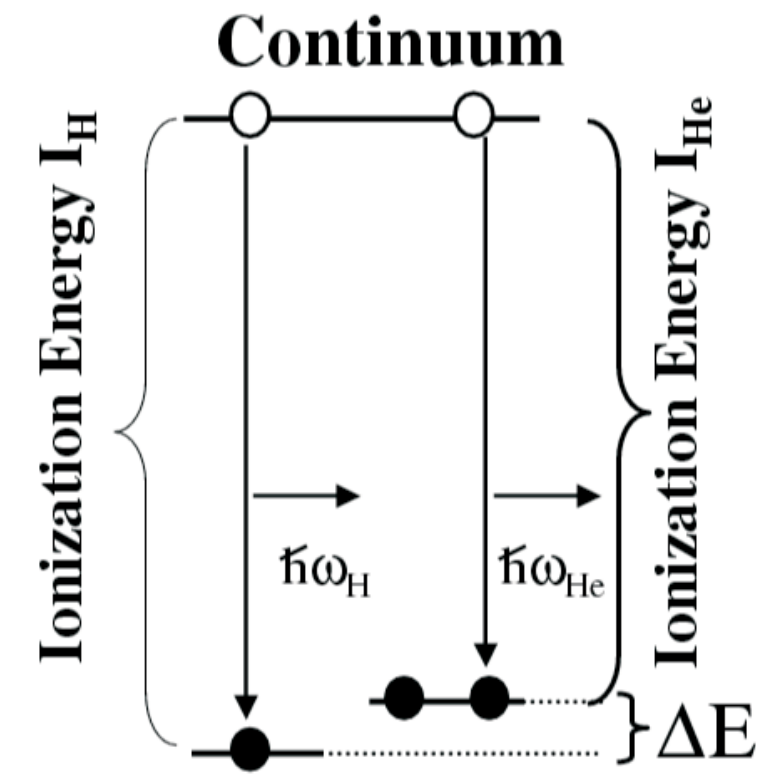

FIG. 1: Schematic presentation of the RR process of free electrons into the initially bare and H-like ions. The energy difference $\Delta E=\hbar \omega_{H}-\hbar \omega_{H e}$ gives exactly the two-electron contribution to the ionization potential in He-like ions.

binding energy in the He-like system. The measurement represents the most accurate determination of two-electron effects in the domain of high-Z He-like ions and reaches the size of the specific two-electron radiative QED corrections. In particular, since the two-electron contributions are experimentally isolated, all one-electron contributions such as the effects of the finite nuclear size cancel out almost completely in this type of experiment. Note, this is an additional distinctive feature of this particular kind of QED test. In contrast, all other atomic-structure experiments at high $\mathrm{Z}$ such as $1 s$ Lamb shift[10-13], bound state $g$-factor [14], hyperfine splitting in hydrogen-like ions [15-18] or 2s-2p transitions in heavy Li-like systems [19-21] encounter the issue of large nuclear effects which may mask the physics at strong fields.

In a series of experiments devoted to the 1 s Lamb shift in H-like uranium, the ESR storage ring has proven to provide unique experimental conditions for high- $\mathrm{Z}$ ions. Here intense beams of cooled bare and H-like uranium are routinely available allowing the extension of former studies at the Super-EBIT to He-like uranium. In contrast to experiments dealing with stationary ions, experiments using stored high-energy beams must deal with large corrections due to the Doppler shift given by $E_{L A B}(\theta)=E_{P R O J} / \gamma(1-\beta \cos \theta)$, where $E_{L A B}$ 


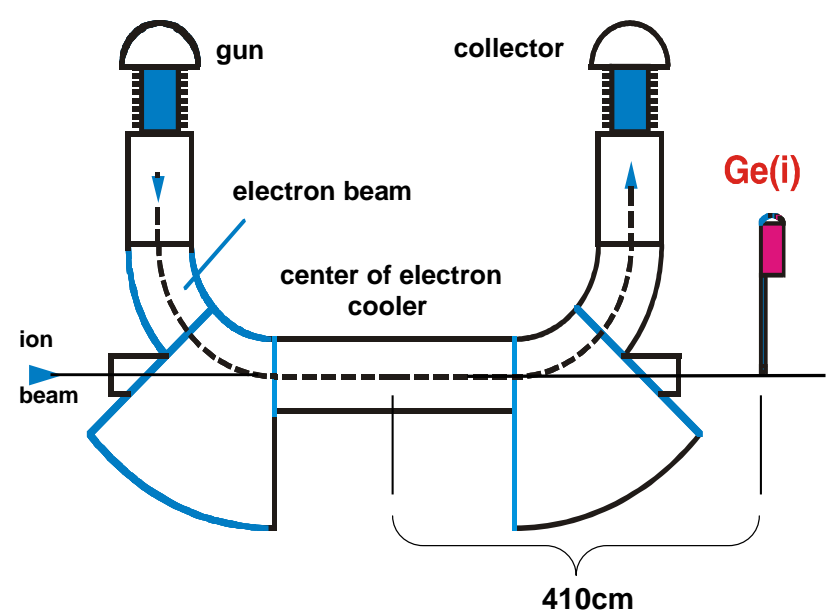

FIG. 2: Scheme of the experimental setup used at the electron-cooler device of the ESR storage ring.

and $E_{P R O J}$ denote the photon energy in the laboratory and in the emission frame, $\theta$ is the observation angle in the laboratory, $\beta$ is the projectile velocity in units of the speed of light, and $\gamma$ is the Lorentz factor. The accuracy in the Doppler shift corrections of $\Delta E / E \leq 10^{-4}$ typically achieved at the ESR is determined by the uncertainty of the absolute velocity. However, for our present study where an energy difference of about $2.2 \mathrm{keV}$ (emitter frame) has to be determined this would introduce an uncertainty of less than $\pm 0.1 \mathrm{eV}$ only.

For the experiment, bare and H-like uranium ions were injected, in alternate order, at the initial energy of close to $360 \mathrm{MeV} / \mathrm{u}$ into the ring and cooled by an electron beam of $300 \mathrm{~mA}$. After the initial accumulation and cooling, the ions were decelerated to the final beam energy of $43.6 \mathrm{MeV} / \mathrm{u}$ using a technique which is routinely available at the ESR. At the final stage of beam handling the electron cooler was switched on again at the energy which corresponds to the energy of the decelerated ions. Here an electron beam current of $100 \mathrm{~mA}$ was used. Applying this procedure typically $2 \times 10^{7}$ ions were decelerated. Moreover, because the key feature of the experiment is a relative measurement of $\mathrm{RR}$ into the K-shell of initially bare and H-like uranium, we changed three times between the two charge states during the experiment. Compared to a simultaneous storage of both species, also possible at the ESR, the method used had the advantage of allowing us to measure the x-ray emission in coincidence with the down-charged ions.

For the experiment, the $0^{\circ} \mathrm{x}$-ray detection apparatus at the ESR cooler section was used, 
an enviroment which was already exploited in great detail in first Lamb-Shift experiments at high-Z by Beyer et al. [12] (a sketch of the experimental setup is displayed in Fig. 2). Here, the recombination photons are produced via radiative recombination of electrons along the two-meter-long straight section of the electron cooler . Since electron cooling results in a constant ion beam energy locked to a value determined by the acceleration voltage of the electron cooler, the merged co-propagating beams of electrons and ions exhibit identical velocities. The longitudinal and transverse temperature amount to $\approx 10^{-4} \mathrm{eV}$ and $\approx 0.1 \mathrm{eV}$, respectively. The absolute beam velocity $\beta$ was derived using the relation $(\gamma-1) m c^{2}=e U_{e}$ where $e$ and $m c^{2}$ are the charge and the rest energy of the electron, respectively. $U_{e}$ is derived from the applied cooler voltage $U$ and corrected for the potential depression due to the space charge of the electron beam. It is given by the formula [22] $U_{e}=U-375 \cdot I_{c}[A]$. The second term represents the space charge correction and was determined by a measurement of the Schottky revolution frequency of the circulating beam as function of the cooler current $I_{c}$.

X-ray detection was accomplished using three independent strips of a segmented germanium detector, each furnished with an individual readout. The horizontal and vertical size of each segment is $13 \mathrm{~mm}$ and $25 \mathrm{~mm}$, respectively with an effective crystal thickness of $12 \mathrm{~mm}$. The detector was mounted on a movable support, $4100 \mathrm{~mm}$ down-stream the center of the electron cooler. Periodically, after the accumulation procedure was finished, the detector was positioned at a perpendicular distance of just $(10 \pm 1) \mathrm{mm}$ from the circulating beam. For the three strips used in the experiment this position corresponds to a mean observation angle of $0.35^{\circ}, 0.53^{\circ}$, and $0.71^{\circ}$ respectively with an angular acceptance of $\pm 0.17^{\circ}$ (all values given refer to the center of the electron cooler). The resulting accuracy of the observation angle $\Delta \theta$ amounts to $\Delta \theta=0.02^{\circ}$.

A very important aspect of the present experiment was an accurate determination of the x-ray energies. Although the intrinsic linewidth of the Ge(i) detector for the energy range of relevance is about $700 \mathrm{eV}$, the small energy difference between two closely spaced lines can be determined with high accuracy [23]. In order to take advantage of this property, a projectile energy of $43.59 \mathrm{MeV} / \mathrm{u}$ was chosen. At this particular beam energy the Doppler shift close to $0^{\circ}$ allowed us to park the $177.214 \mathrm{keV} \gamma$-ray line of ${ }^{169} \mathrm{Yb}$, used for calibration, just in between the K-RR lines for H- and He-like uranium. This is shown in Fig. 3, where the observed X-ray spectra for capture into bare and H-like uranium ions are shown together with the calibration spectrum. Here, we have to add that in order to gain control over 


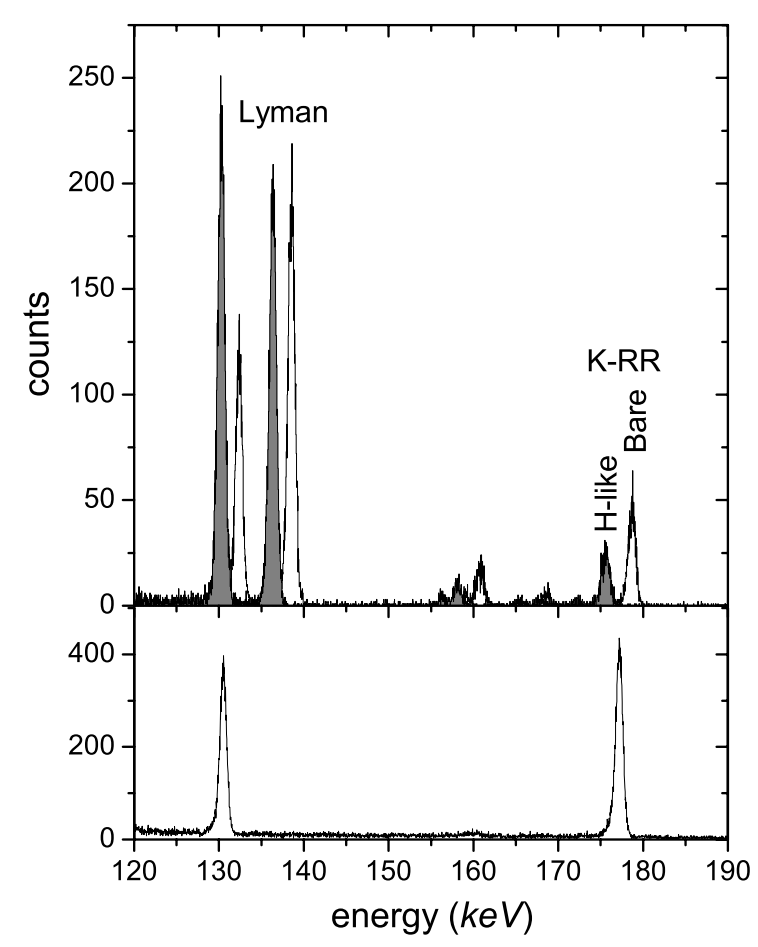

FIG. 3: Top part: X-ray spectra for capture into bare and H-like uranium (forming H-, and He-like uranium respectively) as measured at ESR electron cooler; Bottom part: Spectrum of the ${ }^{169} \mathrm{Yb}$ source used for energy calibration. The two peaks shown are the $\gamma$-ray lines with energies of 130.524 and $177.214 \mathrm{keV}$, respectively.

possible electronic drifts the detector was regularly calibrated, in two to four hour intervals.

The x-ray spectra for each detector segment were recorded event-by-event in coincidence with the events derived from the particle detector. For this purpose Tennelec TC244 NIM amplifiers and 8k ORTEC AD413A CAMAC ADC modules were used. The data analysis closely followed the one discussed in Ref. [9]. The data were divided into individual groups and were analyzed separately. The RR line centroid positions were determined from leastsquares fits using a Gaussian peak shape with a shelf on the low energy side [23]. Since, the $\mathrm{RR}$ lines for the bare and the H-like ions were measured in individual runs, the RR centroid energies were always determined relative to the closely spaced $177.214 \mathrm{keV} \gamma$-ray line of ${ }^{169} \mathrm{Yb}$. Using the $177.214 \mathrm{keV}$ calibration line as a reference, the relative energy separation for RR into the bare and H-like ions were determined for each detector segment separately, 
to $3059 \pm 22 \mathrm{eV}, 3029 \pm 17 \mathrm{eV}, 3098 \pm 36 \mathrm{eV}$ [24]. This corresponds to a weighted mean value of $3047.91 \pm 12.6 \mathrm{eV}$. Finally, from the cooler-voltage reading of $23.924 \mathrm{kV}$ and from the space-charge correction at $100 \mathrm{~mA}$, an effective cooler voltage of $23.913 \mathrm{kV}$ is derived (compare Eq. [2]). This acceleration voltage implies a value of $\beta=0.29565$ with an uncertainty of $5.8 \cdot 10^{-6} \Delta U_{e}$ where $\Delta U_{e}$ refers to the accuracy achieved in the calibration of the cooler voltage $[22,24]$. In our experiment $\left(U_{e}=23.913 \mathrm{kV}\right) \Delta U_{e}$ is given by $\pm 5 \mathrm{~V}$ and we obtain $\Delta \beta= \pm 2.9 \cdot 10^{-5}$. Accordingly (see Eq. 1) the Doppler correction factor is determined to 0.737309 for the first strip, 0.737317 for the second and 0.737327 for the third strip, with an uncertainty introduced by $\Delta \beta$ of less than $\pm 3.2 \cdot 10^{-5}$. The latter is the same for all the three different strips and corresponds to an uncertainty of $\pm 0.071 \mathrm{eV}$ on an absolute scale. The uncertainty caused by $\Delta \theta$ is more than one order of magnitude smaller. Here we like to emphasize, that because of the combination of the $0^{\circ}$ geometry and the deceleration technique the systematic uncertainties introduced by $\Delta \theta$ and $\Delta \beta$ do not affect the final accuracy achieved. In contrast, the result is entirely limited by counting statistics. Therefore, from a Lorentz transformation into the emitter frame, we obtain a value of $2248 \pm 9 \mathrm{eV}$ for the two-electron contribution to the ground state ionization potential in He-like uranium.

\begin{tabular}{|c|c|c|c|c|c|c|c|c|}
\hline Nuclear & 1-photon & 2-photon exchange & 2-photon exchange & 2eSE & $2 \mathrm{eVP}$ & $\geq 3$-photon & Total & Exp. \\
Charge & exchange & non-QED & QED & & & exchange & theory & \\
\hline \hline 83 & $1897.56(1)$ & $-10.64(1)$ & $-0.30(1)$ & -6.73 & 1.55 & $0.06(7)$ & $1881.50(7)$ & $1876 \pm 14$ \\
92 & $2265.88(1)$ & -12.09 & -0.79 & -9.78 & 2.63 & $0.06(9)$ & $2245.92(9)$ & $2248 \pm 9$ \\
\hline
\end{tabular}

TABLE I: Comparison of our experimental result for $Z=92$ with the calculations of Yerokhin et. al. [3] and the result from the Super-EBIT [9] at $Z=83$. All energies are given in $\mathrm{eV}$.

The experimental result obtained for the two-electron contribution is given in table 1 in comparison with the theoretical calculations of Yerokhin et al. [3]. In addition we compare with the experimental result obtained for $\mathrm{Bi}(\mathrm{Z}=83)$ in a former experiment at Super-EBIT [9]. From the table an excellent agreement between our experimental result and the theoretical prediction can be stated. This theory takes into account the electron-electron interaction complete to second order in $\alpha$ [3]. Beyond the first-order one-photon-exchange contribution it also comprises the non-QED contribution of the two-photon exchange as well as the QED 
two-photon exchange part also called the box and the ladder diagram. Most important, the radiative two-electron QED contributions are considered to second order in $\alpha$ in a complete fashion. This two-electron Lamb shift comprises both the two-electron self energy ( self energy screening diagrams) and the two-electron vacuum polarization (vacuum polarization screening diagrams) [3]. Note, that compared to the QED calculations for high-Z H-like systems, where some higher-order QED effects are still uncalculated, the claimed theoretical uncertainty for the two-electron QED contribution is very small and, for the particular case of He-like uranium, estimated to be of the order of $0.1 \mathrm{eV}$ only. Also, we have to add that the theoretical treatment of Yerokhin et al. [3] is in excellent agreement with a further theoretical approach by Persson et al. [2], based on relativistic many-body perturbation calculations, which also comprises all specific two-electron QED effects to second order in $\alpha$. Most importantly, as has been shown by Persson et al. [2] and Yerokhin et. al. [3], these specific two-electron QED effects are almost completely unaffected by the uncertainties of the nuclear-charge radius, one of the most serious limitations for the QED tests in high$\mathrm{Z}$ one-electron systems. As can be deduced from the experimental and theoretical results presented in the table the experimental data provide a meaningful test of the many-body non-QED part of the electron-electron interaction. In particular, the accuracy of the present experiment has reached the size of the two-electron self energy contribution, i.e. of an $\alpha^{2}$ radiative correction.

In summary, radiative recombination transitions into the ground state of cooled bare and hydrogenlike uranium ions were studied at the storage ring ESR, allowing for a direct measurement of the electron-electron contribution to the ionization-potential in He-like uranium. Compared to former experiments at Super-EBIT, this experiment allowed us to extend our knowledge about the electron-electron interaction in the ground state of He-like ions up to the heaviest stable element, i.e. ${ }^{238} U^{90+}$ whereby the sensitivity to the specific two-electron QED effects of $\alpha^{2}$ could be improved by a factor of two. The experiment yields the most accurate determination of two-electron effects in the domain of high-Z He-like ions and reaches already the size of the calculated specific two-electron self-energy correction. In contrast to the H-like ions at high $\mathrm{Z}$, only a moderate improvement of the already achieved accuracy is required to provide a measurement of higher-order QED effects in the absence 
of nuclear size corrections.

[1] P. J. Mohr, G. Plunien, and G. Soff, Phys. Rep. 293, 227 (1998).

[2] H. Persson, S. Salomonson, P. Sunnergren, I. Lindgren, Phys. Rev. Lett. 76, 204 (1996).

[3] V.A. Yerokhin, A.N. Artemyev, V.M. Shabaev, Phys. Lett. A 234, 361 (1997).

[4] G.W. Drake, Can. J. Phys. 66, 586 (1988).

[5] W.R. Johnson and J. Sapirstein, Phys. Rev. 446, R2197 (1992).

[6] M.H. Chen, K.T. Cheng, and W.R. Johnson, Phys. Rev. A47, 3692 (1993).

[7] D.R. Plante, W.R. Johnson, and J. Sapirstein, Phys. Rev. A49, 3519 (1994).

[8] V.M. Shabaev, A.N. Artemyev, and V.A. Yerokhin, Physica Scripta T86, 7 (2000).

[9] Marrs, R.E., Elliott, S.R. and Stöhlker, T., Phys. Rev. A 52, 3577 (1995).

[10] J.P. Briand, P. Chevallier, P. Indelicato, K.P. Ziock, D.D. Dietrich, Phys. Rev. Lett. 65, 2761 (1990).

[11] Th. Stöhlker, P.H. Mokler, K. Beckert, F. Bosch, H. Eickhoff, B. Franzke, M. Jung, T. Kandler, O. Klepper, C. Kozhuharov, R. Moshammer, F. Nolden, H. Reich, P. Rymuza, P. Spädtke, M. Steck, Phys. Rev. Lett. 71, 2184 (1993).

[12] H.F. Beyer, IEEE Trans. Instrum. Meas. 44 (1995), 510; H.F. Beyer, G. Menzel, D. Liesen, A. Gallus, F. Bosch, R.D. Deslattes, P. Indelicato, Th. Stöhlker, O. Klepper, R. Moshammer, F. Nodeln, H. Eickhoff, B. Franzke, and M. Steck, Z. Phys. D 35, 169 (1995).

[13] Th. Stöhlker, P.H. Mokler, F. Bosch, R.W. Dunford, B. Franzke, O. Klepper, C. Kozhuharov, T. Ludziejewski, F. Nolden, H. Reich, P. Rymuza, Z. Stachura, M. Steck, P. Swiat, and A. Warczak, Phys. Rev. Lett. 85, 3109 (2000).

[14] H. Häffner, T. Beier, N. Hermanspahn, H.-J. Kluge, W. Quint, S. Stahl, J. Verdu, G. Werth Phys. Rev. Lett. 85, 5308 (2000).

[15] I. Klaft, S. Borneis, T. Engel, B. Fricke, R. Grieser, G. Huber, T. Kühl, D. Marx, R. Neumann, S. Schröder, P. Seelig, L. Völker, Phys. Rev. Lett. 73, 2425 (1994).

[16] P. Seelig, et al., Phys. Rev. Lett. 81, 4824 (1998).

[17] J.R. Crespo López-Urrutia, P. Beiersdorfer, D. W. Savin, and K. Widmann, Phys. Rev. Lett. 77, 826 (1996).

[18] P. Beiersdorfer, S. B. Utter, K. L. Wong, J. R. Crespo Lopez-Urrutia, J. A. Britten, H. Chen, 
C. L. Harris, R. S. Thoe, D. B. Thorn, E. Träbert, M. G. H. Gustavsson, C. Forssen, A.-M. Martensson-Pendrill, Phys. Rev. A 64, 032506 (2001).

[19] J. Schweppe, A. Belkacem, L. Blumenfeld, N. Claytor, B. Feinberg, H. Gould, V.E. Kostroun, L. Levy, S. Misawa, J.R. Mowat, P.H. Prior, Phys. Rev. Lett. 66, 1434 (1991).

[20] P. Beiersdorfer, A.L. Osterheld, J.H. Scofield, J.R. Crespo Lopez-Urrutia, K. Widmann, Phys. Rev. Lett. 80, 3022 (1998).

[21] D. Feili, Ph. Bosselmann, K.-H. Schartner, F. Folkmann, A. E. Livingston, E. Träbert, X. Ma, P. H. Mokler, Phys. Rev. A 62, 022501 (2000).

[22] M. Steck, private communication (2002).

[23] R.G. Helmer, R.C. Greenwood,R.C. Gehrke, Nucl. Instrum. Methods 124, 107 (1975).

[24] A. Gumberidze, PhD thesis, University of Frankfurt (2003). 